\title{
LAS POLÍTICAS DE LOS EE.UU. EN LA REGIÓN ASIA- PACÍFICO ENTRE 2008-2018: DEL G2 HACIA LA NUEVA CONTENCIÓN ${ }^{1}$
}

\author{
US POLICIES IN THE ASIA-PACIFIC REGION BETWEEN \\ 2008-2018: FROM G2 TO NEW CONTAINMENT
}

\section{Sthefany Rodríguez Rincón ${ }^{2}$ \\ ORCID: 0000-0002-1236-451X}

\author{
Dusan Praj ${ }^{3}$ \\ ORCID: 0000-0002-3676-530X
}

\section{Jorge Andrés Acosta Strobel ${ }^{4}$ \\ ORCID: 0000-0002-3801-418X}

\section{RESUMEN \\ El presente trabajo tiene como objetivo ana- lizar las políticas de Estados Unidos hacia Asia- Pacífico entre el 2008 y el 2018, principalmente la transición de la cooperación hacia la conten- ción de Estados Unidos contra China. Luego de la victoria de Barack Obama en las elecciones presidenciales en EEUU, la política hacia la región de Asia Pacífico pasó por importantes transiciones. El proyecto inicial de la coopera- ción con el nombre de G2 fue reemplazado por}

un giro geoestratégico bajo el nombre "Pívote hacia Asia", el cual englobaba varios ejes de la proyección: el económico, en la forma de Acuerdo Transpacífico y el reforzar alianzas militares como el Diálogo Cuadrilateral de Seguridad. Con este nuevo lema, los Estados Unidos impulsaron distintas alianzas en la región, con el propósito de contrabalancear la creciente influencia de China en la zona de Asia-Pacífico y el aumento de la participación en las disputas territoriales en el Mar del Sur de China.

1 Este artículo de revisión es producto del proyecto de la investigación "De seguridad nacional a la seguridad multidimensional: Transformación y nuevos desafíos para la seguridad en el siglo XXI", adscrito a la Facultad de Estudios Internacionales de Institución Universitaria Esumer y desarrollado durante el año 2020.

2 Negociadora internacional de la Institución Universitaria Esumer, Medellín, Colombia, correo: sthefany.rodriguez@hotmail.com

3 MA in International Relations, University of Comenius, Bratislava - Eslovaquia. Docente a tiempo completo de la Facultad de Estudios Internacionales de la Institución Universitaria Esumer. Correo electrónico: dusan.praj@esumer.edu.co

4 Profesional en Negocios Internacionales y especialista en Legislación Aduanera de la Institución Universitaria Esumer. Estudiante de Especialización en Paz y Desarrollo Territorial - Corporación Unificada Nacional (CUN). Magíster en Gestión en Ciencia, Tecnología e Innovación de la Universidad de Antioquia. Docente investigador, líder del Semillero de Innovación y Emprendimiento de la Institución Universitaria Esumer. Correo: jorge.acosta@esumer.edu.co 
Palabras clave: Estados Unidos; China, Asia-Pacífico; Nueva Contención; Pívot hacia Asia; Geopolítica.

\section{ABSTRACT \\ The aim of this paper is to analyze the poli- cies of the United States towards the Asia-Pacific region, between 2008 and 2018, mainly the US containment strategy against China. After the victory of Barack Obama as president of the Uni- ted States, the United States policy towards this region experienced several changes. The former cooperation project named $\mathrm{G} 2$ was replaced by a}

geostrategic proposal called "Pivot". This proposal changed, both the United States politics and economics in this region by being involved in initiatives as the TPP and military alliances as the Quadrilateral Security Dialogue. China interprets this proposal as a containment strategy due to United Stated alliances with other countries in the region and the constant intervention of this country in the territorial disputes in the South China Sea.

Keywords: United States; China; Asia-Pacific; New Containment, Pivot to Asia; Geopolitics.

\section{Introducción}

Las relaciones tanto políticas como económicas entre China y Estados Unidos constituyen gran parte de lo que define el sistema internacional del siglo XXI. Ante el crecimiento del gigante asiático a nivel comercial y su aumento en capacidad económica y militar, Estados Unidos ha generado políticas variadas que van desde la cooperación hasta la contención de un rival en esfera internacional. El auge de China manifestado por el crecimiento económico sostenido y el aumento constante de presupuesto militar representa el mayor desafío a la posición de los Estados Unidos en el siglo XXI en el sistema internacional.

A partir de lo anterior, la presente investigación pretende determinar las iniciativas en las relaciones entre China y Estados Unidos en los años 2008 - 2018 para, posteriormente, analizar las estrategias políticas que ha implementado Estados Unidos en cuanto a la contención de China por medio de sus aliados en la región de Asia-Pacífico.

Este texto se dividirá en dos epígrafes, los cuales pretenden dar respuesta al objetivo de investigación. En primer lugar, se tomó una temporalidad para la investigación de 10 años, desde 2008 hasta 2018. A partir de esta fecha se describen las políticas de dos gobiernos de Estados Unidos que han estado en el poder (Barack Obama y Donald Trump) hacia Asia-Pacífico. De igual forma, se analizan algunos tratados como el Acuerdo Transpacífico (Transpacific Parternship o TPP), alianzas como Quadrilateral Security Dialogue (QSD) que forman la iniciativa del Pívote hacia Asia, el incremento de actividad militar en la zona y cómo China responde a las políticas de contención. Se mencionan temas como el fortalecimiento de nuevas alianzas estadounidenses con diferentes países de la región Asia-Pacífico; así como las disputas que se están dando en el Mar del Sur de China y las respuestas sobre la posición que ha tomado el gigante asiático. 
Bustelo (2004) expone las manifestaciones por parte de China donde explica el crecimiento del gigante asiático como un Estado emergente y cómo puede llegar a generar conflictos con los intereses estratégicos de Estados Unidos.

El escenario global ha cambiado sustancialmente en los primeros 10 años del siglo XXI y lo que ha empezado a sentirse fue una disminución en la posición de los Estados Unidos, la cual aceleró posterior a la crisis financiera del 2008 con la consolidación de los BRICS (grupo de países emergentes compuestos por Brasil, Rusia, India, China y Sudáfrica) como una expresión de la independencia de los intereses estadounidenses, al menos en dicha época (Castro, Acosta, \& Praj, 2019).

Por otra parte, se ha evidenciado un crecimiento en la capacidad militar de China, con cada vez más importancia para el desarrollo de su política exterior, así como un aumento de las tensiones en los conflictos regionales que afectan la estabilidad geopolítica, tal cual sucede en el Mar del Sur de China (Mijares, 2006). Ante esto, la presencia de Estados Unidos en la región de Asia-Pacífico se intensificó desde el gobierno de Barak Obama, durante el cual esta región se convirtió en el eje estratégico de planeamiento de los Estados Unidos, utilizando su influencia en la región e implementando políticas de seguridad con los Estados pertenecientes al continente asiático (Manyin et al., 2012).

Con respecto a los temas de investigación anteriores, Praj \& Restrepo (2017) establecen los nuevos desafíos en la geopolítica actual con respecto a China, las disputas que se están presentando en el Mar del Sur de China y nuevos proyectos como la Nueva Ruta de la Seda. Ante esto, se evidencia que la geopolítica China está cambiando las relaciones en el Pacífico asiático y no solo hay mayores movimientos, sino que se puede observar el fortalecimiento militar y la creación y fortalecimiento de alianzas entre los Estados Unidos y diferentes países asiáticos.

\section{Marco teórico}

Para este artículo es oportuno aclarar algunos de los conceptos teóricos de vital importancia para el abordaje realizado desde las diferentes corrientes teóricas de las Relaciones Internacionales. A partir de este contexto, se hará un enfoque desde el realismo, que mira los aspectos del poder y seguridad internacional. 


\subsection{Realismo}

El realismo plantea la lucha constante por el poder entre países en un marco internacional. Estas naciones batallarán según los intereses en los cuales sus políticas están puestas, su objetivo principal es obtener poder, para así consolidarse como entidades autónomas y establecer las relaciones de seguridad. En esta visión el poder no es equilibrado, por lo que existen países con mayores grados de poder que predominan a nivel global.

Dougherty y Pfaltzgraff (1971) plantean que los Estados con mayor capacidad militar para defenderse de algún ataque proveniente de un rival son los que prevalecerán. La lucha persistente por el poder trae consigo el dilema de seguridad constante, en el cual el aumento de la seguridad de un Estado implica, automáticamente, la amenaza a la seguridad del otro. Ante esto, Pérez (1996) indica que la supervivencia de los Estados en el sistema internacional se divide en tres lineamientos: (i) los Estados temen a otros Estados, (ii) cada Estado intenta garantizar su propia supervivencia y, por último, (iii) los Estados intentan maximizar su posición de poder sobre otros Estados

A partir de esto se despliegan dos conceptos que son importantes tener en cuenta al momento de hablar de realismo, estos son: poder duro y poder blando; pero para poder entender la idea de poder es importante conocer su definición.

\subsection{Poder}

Nicolás Creus define el poder (2013) como algo que no puede ser medido rigurosamente, esto se debe a los múltiples comportamientos que existen. Desde la perspectiva de las relaciones internacionales, se puede definir el poder como el potencial que tiene una nación de imponer su voluntad sobre otra, además de nombrarlo como "una relación entre hombres".

Ante esto, Hans Morgenthau (2003) define el poder como el control del ser humano sobre las mentes y acciones de otras personas; en este sentido, es importante traer la visión de Pier Paolo Portinario (2000) que precisa que el poder es la utilización de la fuerza para contribuir a diferentes efectos en un ambiente determinado. De igual forma, define que el poder termina ejerciéndose ser humano sobre ser humano. Así, establece que una de las partes siempre generará cambios en la otra a través del poder. Indica, también, que no hay poder sin el acceso a recursos por uno de los implicados y la ausencia del otro en ese proceso. 
Así, a partir de estas definiciones, se podrá tener un panorama más amplio para los conceptos de poder blando y duro. Joseph Nye (2004), relaciona el poder duro como "un empujón" mientras que el poder blando lo aproxima a "la atracción". Praj (2018) anota que el poder duro se compone por recursos que son tangibles, como, por ejemplo: el PIB, la población, territorio, geografía, recursos naturales, tamaño y equipamiento de ejército; en pocas palabras, en aspectos medibles. Mientras que el poder blando se le atribuye a todo aquello intangible, por ejemplo: política exterior, valores políticos, educación, innovación, cultura. En este orden de ideas, poder duro se define como la habilidad de una de las partes para influir, por cualquier tipo de medio, sobre otro de los actores; mientras que el poder blando se adhiere a esas influencias, debido a que se tiene un pensamiento de poder "con" en lugar de "sobre".

\section{Metodología}

Desde un enfoque cualitativo, se buscó dar solución al problema de investigación mediante el rastreo de fuentes secundarias mediante la revisión documental. Se analizan documentos de carácter académico de repositorios, así como libros, artículos científicos, entre otros.

Para la recolección de los datos se tuvieron en cuenta fuentes entre 2008 y 2018, con el apoyo bases de datos como EBSCO, SCOPUS y DIALNET. Lo anterior, con el propósito de indagar el crecimiento de China como potencia emergente y cómo ha sido el accionar de los Estados Unidos para el fortalecimiento de los países de la región Asia Pacífico. La metodología fue de corte analíticodescriptiva y en esta se consideraron, también, medios especializados como The Diplomat, así como fuentes periodísticas como la BBC y DW.

\section{Resultados}

Para entender las iniciativas tomadas por parte de los Estados Unidos para contrarrestar la influencia de China en la región de Asia-Pacífico es importante recalcar puntos que han sido coyunturales en las relaciones políticas y económicas de ambos países. Para esto se tomará como punto de partida los dos mandatos presidenciales en Estados Unidos durante el periodo 2008 - 2018, lapso en el que han ocurrido cambios sustanciales en las políticas estadounidenses hacia esta región del mundo. 


\subsection{Relaciones entre Estados Unidos y China durante el mandato de Barack Obama (2009 - 2017)}

Los ataques terroristas ejecutados por el grupo Al Qaeda el 11 de septiembre de 2001 representaron un golpe al prestigio de Estados Unidos. Como reacción, el gobierno de George W. Bush (2001-2009) decidió intervenir militarmente en Afganistán (2001) e Iraq (2003). El estancamiento político-militar en dichos conflictos vino con la crisis económica de 2008, que trajo la consolidación de los BRICS (2008) como nuevo bloque emergente y donde se evidenció una pérdida de preponderancia del Estado norteamericano y el surgimiento de nuevos actores internacionales (Acosta, Londoño, \& González, 2015; Castro, Acosta, \& Praj, 2019).

El sucesor de George Bush, Barack Obama, decidió reconfigurar el compromiso de Estados Unidos en ambos conflictos, debido a los costos humanos y económicos y la caída de la popularidad de las intervenciones en el extranjero y, también, por incapacidad de presentar una victoria política en los posconflictos de Iraq y Afganistán. La retirada de tropas estadounidenses de Medio Oriente, junto al crecimiento del gigante asiático, generaron una redirección del interés geoestratégico de los EE.UU. hacia Asia Pacífico (Ahumada, 2008).

El crecimiento económico de China, sostenido por más de 30 años consecutivos con un promedio de $10 \%$ anual, ha tenido como consecuencia la transformación del país asiático no solo como una potencia económica, sino cada vez más, también, como potencia militar. Lo anterior está representando el mayor cambio en el entorno geopolítico global. Hay que añadir que China ha incrementado desde 1990 hasta 2017 su gasto militar y es el segundo país con mayor inversión militar a nivel global, solo por debajo de Estados Unidos (The China National Statistics Yearbook, 2017) (Ver gráfica 1).

Según los datos del Instituto de Estudios para la Paz de Estocolmo (2018), China se encuentra entre los países con mayor incremento relativo del gasto en defensa entre 2008 y 2017, junto con Camboya (+332\%), Bangladesh $(+123 \%)$ e Indonesia $(+122 \%)$. El dato actual para China corresponde a $(+110 \%)$.

Los países ubicados en Asia Pacífico percibieron el aumento de gasto militar chino con preocupación, al buscar un equilibrio del poder para la región. Sin embargo, Estados Unidos no se quedó al margen y durante el mismo tiempo ha buscado tener cercanía con la región (Giné, 2010). En este sentido, Bassets (2012) indica un nuevo enfoque de los Estados Unidos hacia la nación china, que ha generado un cambio de orientación denominado como Pivote hacia Asia 


\section{Gráfica 1. El aumento del presupuesto militar chino (1990-2017)}

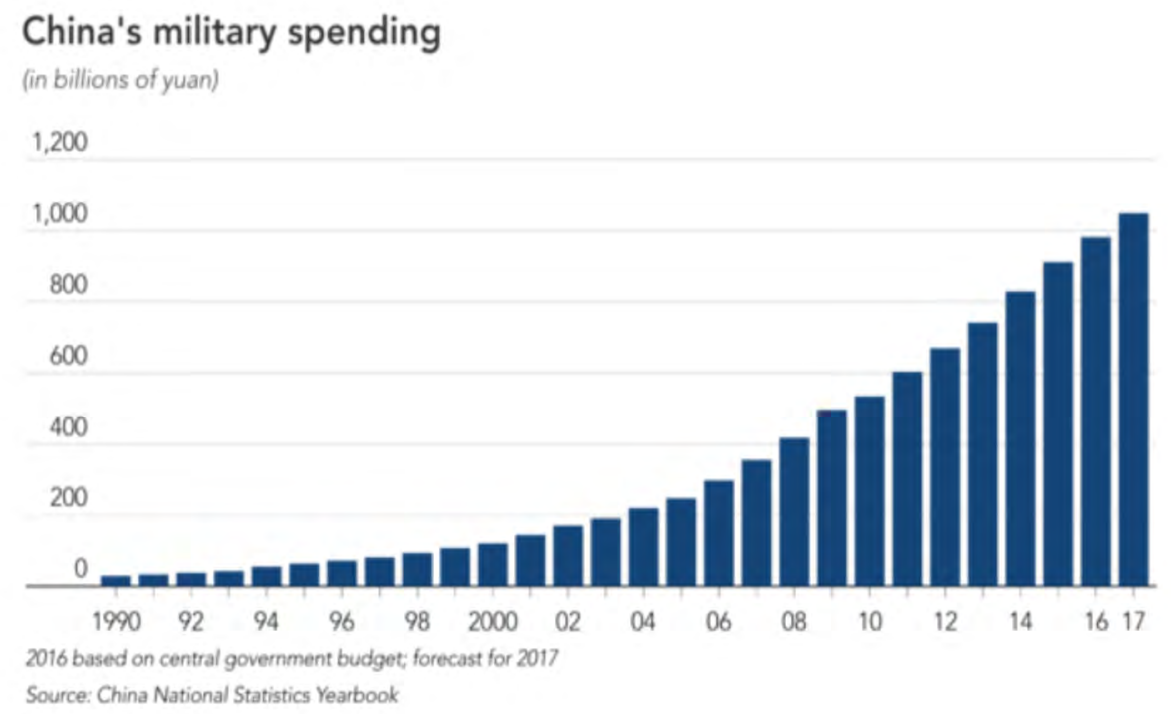

Fuente: The China National Statistics Yearbook (2017).

y concebido como un giro desde las zonas de interés de la estratégica tradicional de los EE.UU., como Oriente Medio y Europa, hacia Asia y también un redespliegue de la política exterior estadounidense desde las cuestiones de terrorismo y conflictos asimétricos hacia los asuntos de la competencia global; desde las viejas naciones aliadas hacia el mundo emergente, o incluso, desde el unilateralismo hacia la cooperación, lo cual ha generado una reformulación de liderazgo de Estados Unidos.

En este orden de ideas, el gobierno de Barack Obama definió el Pivote como un refuerzo de las alianzas bilaterales de la seguridad, el profundizar la cooperación con los poderes emergentes, el compromiso con las instituciones multilaterales regionales, la expansión comercial y de inversiones, la amplia presencia militar y el avance en temas de democracia y de derechos humanos.

Hay que destacar que China aumentó su presencia militar en la zona con objetivos muy claros. El establecimiento del llamado "Collar de perlas chino" se había puesto en marcha a principios del siglo XXI, con el propósito de preservar los flujos de petróleo que iban desde Oriente Medio y África hacia China. Las bases navales chinas establecidas en el Collar de perlas deben servir también 
como bases logísticas para la dimensión naval del proyecto de La Nueva Ruta de la Seda. Estas iniciativas chinas motivaron a los EE.UU. a implementar su propia estrategia, el Collar de contención. En principio, el Collar de contención estadounidense tenía la misma extensión que el actual Collar de perlas chino. En este sentido, la propuesta principal era establecer bases navales para controlar la ruta petrolera de Oriente Medio hacia los puertos chinos. De esta manera, EE.UU. tendría un acceso fácil y rápido para presionar al régimen de Pekín.

Los EE.UU. incorporaron las bases ya existentes en la zona de Asia Pacífico y Oriente Medio para crear dicho Collar con el objetivo de prevenir y balancear la presencia naval china en Indo-Pacífico. La distribución de las bases militares estadounidenses permitió que el cerco de estas quedara con más amplitud que el Collar de perlas chino. Teniendo en cuenta las alianzas de diferentes países de la región Asia Pacífico con EE.UU., la red de bases navales les facilitaría a estos una mayor capacidad y rapidez de despliegue militar del que ahora cuenta China (El Orden Mundial, 2018).

Según Arancón (2014), el collar de contención estadounidense va desde la base surcoreana de Incheon-Osan a través Okinawa, a poca distancia de las disputadas islas Senkaku, la base de Guam; Darwin, en Australia; las islas Cocos, en el Índico; Singapur; la isla de Diego García donde existe un enlace con las bases en Oriente Medio y Asia Central. Yibuti, en el cuerno de África, Yemen, Omán, Bahréin, Qatar, Kuwait, Pakistán con tres bases aéreas, Afganistán, Kirguizistán, Uzbekistán y Turkmenistán.

Además del collar de contención, los EE.UU. empezaron a participar en varias iniciativas estratégicas en la zona de Asia-Pacífico. En el año 2007 inició el Diálogo Cuadrilátero Seguridad- Quadrilateral Security Dialogue (QSD), conformado por Japón, Estados Unidos, India y Australia e impulsado por el premier ministro japonés Shinzo Abe, con el propósito de balancear la creciente influencia de China en la región. De todas maneras, la alianza de cuatro países integrantes de la QSD registró una caída en la actividad en 2008. Las protestas chinas contra los ejercicios navales entre los países de Diálogo con Singapur y las políticas de premier ministro de Australia, Kevin Rudd, implicaban una ruptura temporal en la cooperación entre estos. Además, los últimos cambios políticos en Japón que llevaron al poder al primer ministro Yasuo Fukuda, más abierto a relaciones más estrechas con China, así como también las políticas de primer ministro indio Manmohan Singh, que estableció la relación con China como una prioridad para política exterior de India, han implicado un impedimento al desarrollo de dicha iniciativa. 
Sin embargo, los acuerdos entre Obama y Kevin Rudd y su sucesora Julia Gillard trajeron importantes cambios como el despliegue de las tropas estadounidenses cerca de Darwin en Australia, cerca al Mar del Timor y del Estrecho de Lombok. Al mismo tiempo, los EE.UU., Japón e India se concentraron al conducir los ejercicios navales denominados como Malabar (Rudd, 2019). Cierto rempuje se dio en la cumbre de la ASEAN (Asociación de las Naciones de Sureste Asiático), en 2017, donde se reafirmó la alianza y se acordó priorizar la seguridad en el Mar del Sur de China (Australian Goverment Department of Foreign Affairs and Trade, 2018).

Sin embargo, hay que destacar que las relaciones entre EE.UU. y China no estaban orientadas, desde el inicio, a la contención. En el lapso 2007 - 2010, se propuso un gran proyecto de cooperación internacional bajo el nombre G-2, el cual debía haber incluido Estados Unidos y China. El motivo para proponer el proyecto no era ilógico, en cuanto al contexto económico estas dos naciones representan las dos economías más importantes del mundo. Conforme a lo anterior, Bergsten (2009) determina lo siguiente:

1. China pronto se convertirá en la segunda economía más importante en el mundo detrás de Estados Unidos (se convirtió en 2010).

2. Las dos economías representaban casi la mitad del crecimiento económico mundial en 4 años antes de la crisis financiera 2007/2008.

3. Ambos países son los mayores actores del comercio internacional.

4. Son las dos economías que generan mayor contaminación medio ambiental a nivel global.

5. Están en los polos opuestos del equilibrio comercial y financiero más grande del mundo, Estados Unidos por su parte es el país con mayor déficit y deudas, mientras que China es el país con mayor superávit y el mayor número de reservas monetarias en dólares estadounidenses.

6. Son líderes de dos grupos importantes: Estados Unidos de los países de altos ingresos y altamente industrializados, mientras que China de los mercados emergentes, ambos responsables por más de $50 \%$ de la producción global. 
Todos esos argumentos eran una base sólida para la coordinación efectiva en la política internacional y para una relación más estrecha entre ambas naciones (Sepúlveda, 2012). Ambos países no estaban obligados a estar de acuerdo en todos los puntos de la agenda, por ejemplo, en cuanto a las disputas territoriales del Mar del Sur de China o el impulso de los Estados Unidos por la democracia en Birmania (Pacheco, 2014). Sin embargo, China y Estados Unidos debían trabajar en conjunto de manera constructiva en la política exterior, tanto como en asuntos económicos transcendentales, como la recuperación de la crisis económica del 2008, la agenda de cambio climático y la no proliferación de armas de destrucción masiva, entre otros (Bergsten, 2009).

A pesar de que la cooperación entre los dos líderes internacionales era una idea prometedora, hubo diversas opiniones expertas acerca de la capacidad de Estados Unidos y China para llegar a mutuo acuerdo al momento de la resolución de asuntos particulares (Economy \& Segal, 2009). Una de las mayores preocupaciones para impedir la cooperación eran las relaciones económicas donde predomina el problema de Estados Unidos al tener una balanza comercial crónicamente pasiva con respeto al Estado asiático (Garrett, 2014).

El interés de los Estados Unidos de reforzar su posición económica en la región de Asia-Pacífico encontró su fortalecimiento a través del proyecto de Tratado Transpacífico (conocido como TPP, por sus siglas en inglés), el plan de zona de libre comercio liderado por el Estado norteamericano incluyendo a Japón, Canadá, México, Chile, Perú, Australia, Nueva Zelanda, Singapur, Brunei, Vietnam, Malaysia que representaban $40 \%$ del PIB global, $30 \%$ de exportaciones globales, $25 \%$ de importaciones globales y 793 millones de consumidores para el año 2014. El TPP tuvo por objeto la creación de una plataforma para una potencial integración económica en la región de Asia-Pacífico (Rodríguez, 2014; Sistema de Información sobre Comercio Exterior, 2017).

Para el 2009, el presidente Barack Obama hizo pública la iniciativa con el fin de formar dicho acuerdo regional (Sistema de Información sobre Comercio Exterior, 2013). El presidente norteamericano propuso los siguientes dos ejes:

- Económico. Luego de la crisis económica en el 2008 Estados Unidos venía recuperándose, y la región asiática venía creciendo de manera exponencial, por lo que esto resultaba muy beneficioso para el propio desarrollo.

- Geopolítico. En los países asiáticos, específicamente la región de Asia-Pacífico, se estaba presentando un cambio en cuanto a las relaciones de los países de la región con respeto a crecimiento de China, por lo que cada vez era más latente el riesgo de conflicto entre ellos y, por ende, que se perdiera la estabilidad y la paz entre estos (Green \& Goodman, 2015). 
En este contexto Ajami (2018) señala que el TPP tenía una clara dimensión geopolítica, la cual se basaba en la política del Pivote hacia Asia por parte del gobierno de Barack Obama. En las propias palabras de Obama, los Estados Unidos no pueden dejar a países como China escribir las reglas de la economía global. La exclusión de China, como segunda mayor economía del mundo con el valor combinado de exportaciones e importaciones de 4,3 trillones de dólares para el año 2010 fue una señal muy clara para demostrar que el proyecto tenía la intención de crear y reforzar la zona de influencia de los EE.UU. en Asia Pacífico (Tellis, 2014). Si se hubiera concluido la creación de dicho bloque en las intenciones propuestas por los Estados Unidos, China habría perdido 100 billones de dólares anuales en las exportaciones y perdería 2,2 puntos porcentuales en su PIB, lo que convertiría el TPP en un instrumento económico dominante de la iniciativa del Pivote hacia Asia (Sempa, 2019).

Otra diferencia crucial en relaciones bilaterales entre Estados Unidos y China fue la península de Corea. La amenaza de cohetes balísticos del Corea del Norte llevó al Estado norteamericano y a Corea del Sur, en 2016, a implementar un nuevo sistema de defensa antimisil en Corea del Sur con el nombre THAAD (Terminal High Altitude Area Defense). Sin embargo, dicho paso creó mucha preocupación no solo por parte de Corea del Norte, sino también por parte de China, la cual lo denominó como una amenaza a la seguridad regional. La declaración oficial del gobierno de China consideró la instalación de THAAD como ampliación de la arquitectura de los escudos antimisiles de los EE.UU. en la región de Asia-Pacífico, la cual limita las posibilidades de la disuasión nuclear china y confirma la política de la nueva contención de los EE.UU. hacia esta. Se afirmó que dicho sistema no era eficiente contra los misiles norcoreanos, entonces el verdadero objetivo sea disminuir la capacidad nuclear de China y no proteger de un ataque de Corea del Norte. Además, la instalación de sistema THAAD podría desbalancear las relaciones militares en Asía Pacífico, llevando toda la región a una carrera armamentista.

Para prevenir la instalación del escudo, China tomó pasos de coerción económica contra el gobierno surcoreano. Precisamente, después de anunciar el acuerdo sobre THAAD, los reguladores de comercio del gobierno de China catalogaron algunos artículos de importaciones de Corea del Sur como prohibidos y negaron la ampliación de líneas aerotransportadoras coreanas hacia China. De igual forma, el gigante asiático mermó la venta de paquetes turísticos a la ciudadanía china, que quisieron visitar a la nación surcoreana, lo cual ha representado un golpe duro para el sector turístico del país peninsular, puesto que los grupos turistas de China representaban el $47 \%$ de los grupos viajeros entrantes a Corea del Sur (Meick \& Salidjanova, 2017). Hay que añadir que después de estos pasos, el presidente surcoreano Moon Jae-in, a pesar de instalar dicho sistema, 
buscó mantenerlo inoperante con el argumento de que los misiles balísticos podrían afectar el medio ambiente.

\subsection{Relaciones entre Estados Unidos y China en la presidencia de Donald Trump (2017 - 2018)}

Durante las elecciones presidenciales de Estados Unidos de 2016, existían dos vertientes importantes: seguir con la dinámica de política exterior de Obama a través de Hillary Clinton o generar un cambio en todo el estilo de Gobierno a través de Donald Trump.

Las posturas del mandatario Trump representan un gran giro con respeto a la administración anterior, predominantemente en intención de redefinir algunos acuerdos bi y multilaterales, además de la disminución del apoyo militar a los países aliados con el propósito a motivarlos a participar con más recursos propios militares y económicos en su defensa (Martínez, 2018). Japón paga anualmente 2 billones de dólares como gastos asociados al estacionamiento de 54000 tropas estadounidenses. Donald Trump solicitó el aumento de pago anual a 8 billones de dólares, un aumento de 300 por ciento. En el caso de Corea del Sur, con 28500 tropas estadounidenses estacionadas y con el pago de gastos asociados de 1 billón de dólares anuales, la solicitud de EE.UU. es subir la suma de 50 por ciento, es decir, a 1,5 billones de dólares anuales (Seligman \& Gramer, 2019).

Las dudas sobre la voluntad de EE.UU. de cumplir los compromisos militares hacia sus aliados está creando incertidumbre sobre el futuro y la fuerza de obligación de protegerlos, en otras palabras, resguardar estos Estados bajo la sombrilla nuclear de los EE.UU. en el caso de la amenaza militar vital. Una posible consecuencia podría significar que cada uno de los países tomaría mayor participación en su propia defensa, aumentando considerablemente los gastos militares, desatando carreras armamentistas en la región y creando una arquitectura de seguridad regional alterna con la participación disminuida de los EE.UU., o en los casos extremos, directamente no presente.

En 2016, Donald Trump anunció la salida de los EE.UU. del Acuerdo Transpacífico (TPP); junto con el intento de renegociar también otros tratados comerciales bi- y multilaterales, según él, desventajosos para los EE.UU. (BBC Mundo, 2017). 
La decisión de los EE.UU. de salirse del tratado significó la pérdida de $60 \%$ del PIB del bloque (Ver Tabla 2). Hay que añadir que la salida de los EE.UU. no representó el fin del proyecto, por lo que los países involucrados decidieron seguir en las negociaciones con el mismo nombre sin la participación de los EE.UU., pero en el cual paradójicamente China declaró su deseo de estar incluida (Raffo, García, \& Díaz, 2018).

Tabla 1. Porcentaje de participación de los países al TPP

\begin{tabular}{|c|c|}
\hline Países & $\begin{array}{c}\text { Participación (\% del PIB total del } \\
\text { TPP) }\end{array}$ \\
\hline Estados Unidos & $60,5 \%$ \\
\hline Japón & $17,7 \%$ \\
\hline Canadá & $6,6 \%$ \\
\hline Australia & $5,4 \%$ \\
\hline México & $4,5 \%$ \\
\hline Otros países miembros al & $5,26 \%$ \\
TPP & \\
\hline
\end{tabular}

Fuente: Elaboración propia con base en Sánchez (2017).

No obstante, la reconfiguración del tratado, sin la participación de los Estados Unidos, abrió, de cierta manera, las puertas a China, la cual ha podido tener mayor injerencia en los Estados de la región (Almoguera \& Fontdeglória, 2018). China anunció también su propio proyecto de la integración regional, alterno al TPP, denominado RCEP (Regional Comprehensive Economic Partnership), el cual está incluyendo los países de ASEAN (Asociación de los Países de Sureste Asiático) más naciones como China, Japón, Corea del Sur, Nueva Zelanda, Australia e India. Este proyecto vincula un bloque comercial con mayor número de habitantes y el tercero mayor PIB del mundo. Sin embargo, las preocupaciones de India de estar inundada por los alimentos baratos de China están dificultando la firma final de acuerdo (DW, 2019).

Por otro lado, el gigante asiático presenta el "One Belt, One Road Initiative" (Iniciativa de la Franja y la Ruta) como mayor proyecto de construcción de infraestructura en el continente euroasiático y el AIIB (Banco Asiático de Inversión e Infraestructura), conformados por países tan variados como Arabia Saudita, Bangladesh, Brunéi, Camboya, República Popular China, Corea del Sur, Emiratos Árabes Unidos, Filipinas, India, Indonesia, Irán, Jordania, Kazajistán, entre otros, como instrumento de financiación de las obras. Ambos proyectos están representando una alternativa del Estado chino al modelo de 
globalización liderado por los EE.UU. y al sistema de instituciones financieras internacionales dominado por parte de los Estados occidentales como el Fondo Monetario Internacional y Banco Mundial, con su cuerpo regional, el Banco Asiático de Desarrollo (Praj \& Restrepo, 2017).

Sin embargo, la nueva contención no solo se limita a las iniciativas de la integración económica. Estados Unidos trata de reforzar alianzas con países de la región de Asia-Pacífico, especialmente con naciones como Japón, Corea del Sur, Malasia y Filipinas que se han considerado históricamente como aliados estratégicos (Batalla, 2006; Piqué, 2020).

El punto neurálgico de las relaciones entre Estados Unidos y China es el Mar del Sur de China, que ha cobrado importancia geoestratégica y geopolítica puesto que está separando Asia continental de los Estados insulares de Asia-Pacífico.

El significado de dicho mar demuestra la concentración del volumen de comercio internacional que está pasando por sus aguas. Según el Centro de Estudios Estratégicos e Internacionales, anualmente está pasando 3,37 trillones (miles de billones) de dólares por la zona y son Vietnam (86\%), Indonesia (85\%), Tailandia (74\%), Singapur (66\%) y Malaysia (58\%) los Estados con mayor uso de las rutas marítimas en el Mar del Sur de China (Panda, 2017).

La participación del gigante asiático en el uso de vías marítimas del Mar del Sur de China corresponde a un $39 \%$, esto conllevará a que esta zona acuosa sea determinante para la actividad económica del Estado chino. Además de tener conexiones con Europa y EE.UU., la mayoría de bienes salen de las costas del continente asiático, pero también el ingreso de las materias primas para la fabricación de diferentes productos se da por este mar, por lo tanto, es de importancia para la economía mundial. La ruta del Mar del Sur de China supone una de las rutas más importantes en la región, puesto que representa el $50 \%$ de sus importaciones y exportaciones. Además, el $80 \%$ de las importaciones de petróleo y otras fuentes como gas natural para los países de Asia Pacífico pasan por este mar (Escudero, 2018).

De igual forma, es importante analizar las reservas de petróleo en la zona. Según reporta la Agencia Internacional de Energía (EIA en inglés), en el subsuelo marítimo del Mar del Sur de China existe una reserva de 11000 millones de barriles de crudo y, aproximadamente, 190 billones de pies cúbicos de gas natural (Cuenca, 2018). El mismo informe estima que Vietnam cuenta con 3000 millones de barriles de crudo en el fondo del mar y 20 billones de pies cúbicos de gas natural. Del mismo modo China cuenta con 1300 millones de barriles 
de petróleo y 15 billones de pies cúbicos de gas natural. Mientras que Filipinas, Malasia e Indonesia tienen alrededor de 5500 millones de barriles de crudo y 139 billones de pies cúbicos de gas natural (Actualidad RT, 2016; Cuenca, 2018).

En 2009, con motivo de la petición hecha por Vietnam y Malasia, de ampliar la plataforma continental y ampliar la Zona Económica Exclusiva a 350 millas náuticas, China muestra el mapa denominado "El mapa de los nueve trazos", el cual debe comprobar el derecho de China a alrededor del $80 \%$ del Mar del Sur de China y pide su reconocimiento por la comunidad internacional. Adicionalmente, con respecto a la petición por parte de Vietnam, el gobierno chino sostiene que dicha demanda viola la soberanía del país y que, por lo tanto, es un acto ilegal e inválido. Con este mapa no reclama solo su "derecho histórico", sino que restringe que los demás países costeros tengan acceso a las zonas económicas exclusivas respectivas (Observatorio de la Política China, 2009).

China, para proteger sus intereses territoriales, aplica la estrategia denominada A2/AD (anti access/area denial, traducida al español como la negación de acceso y prohibición de área) para las tripulaciones navales y aéreas militares de Estados Unidos, con el objetivo de mantener las tropas estadounidenses lejos de zonas estratégicas del gigante asiático, incluyendo los territorios disputados en el Mar del Sur del China.

Ante la creación de islas artificiales como plataformas de ubicación de sistemas del reconocimiento e identificación aérea y naval, junto con los sistemas de la defensa antiaérea y misiles antibuques por parte de China, se han generado tensiones con Estados Unidos y otras naciones ubicadas en la zona, las cuales se encuentran afectadas ante las políticas de expansión del gigante asiático (Minh Tri, 2017). El grupo de islotes de Paracelso y Spratly, la razón original de las demandas territoriales de China en la zona, se ha vuelto crucial: aquella nación que adquiera estos islotes podrá redefinir su zona económica exclusiva y todos los recursos pertenecientes a esta (Vuving, 2016).

Además, la explotación de las reservas submarinas de hidrocarburos escaló el choque entre China y sus vecinos (Vargas, 2019). La República Popular de China creó plataformas petrolíferas en aguas territoriales de Vietnam en 2014, que se ha negado a explotar recursos petroleros de manera conjunta con el gigante asiático. Como retaliación, China ha impedido la pesca dentro de la zona, que a su vez ha generado más conflictos con el Estado vietnamita. Eso ha derivado en múltiples manifestaciones sociales, incluyendo la expulsión de personas chinas y daños a algunas empresas chinas presentes en Vietnam (BBC News, 2014; Whipple, 2017). 
Según David Shear (secretario adjunto de defensa norteamericano), los Estados Unidos, así como cada uno de los Estados, tienen derecho a libre navegación en la región. Por otra parte, Estados Unidos ha buscado tener la presencia en la zona mediante el despliegue de portaviones y navíos de combate en la región. Además, de promulgar el principio de libre navegación, el Estado norteamericano considera que las islas artificiales chinas son una amenaza para seguridad regional, sin contar que también crean un área de alto control de esta zona (Bustelo \& Soto, 2003; Stashwick, 2018).

Otros países como Filipinas, Malasia, Vietnam y Brunei participan en dichas disputas porque las demandas territoriales chinas afectan sus fronteras marítimas. Estados Unidos, en el intento de contrarrestar las ambiciones de China en el Mar del Sur de China, está profundizando las alianzas tradicionales con Filipinas. Además, están tratando de crear una nueva arquitectura de las alianzas regionales impulsada por la preocupación que genera la actuación de China como con Malasia, donde el presidente Obama hizo la primera visita de un presidente estadounidense desde la visita de Lyndon B. Johnson en 1966. La visita de Obama en 2014 fue motivada, precisamente, como demonstración del interés de los Estados Unidos en la zona de Asia-Pacífico frente al auge de República Popular en su propio patio trasero (Mandelbaum, 2019). Los EE.UU. también proporcionaron 26,25 millones de dólares a Vietnam dentro de la Iniciativa de Seguridad Marítima de Sureste Asiático para reforzar la capacidad de protección de sus zonas marítimas y 56 millones de dólares como asistencia bilateral de seguridad como Financiación Militar Foránea (Dalton, 2020). Sin embargo, el giro actual hacia aislacionismo por los EE.UU. deja los aliados en gran incertidumbre frente a las aspiraciones geopolíticas por parte del Estado chino.

\section{Discusión y conclusiones}

Debido al inminente resurgimiento de China en términos económicos, militares y geoestratégicos, Estados Unidos ha hecho un giro militar, económico y político hasta esta región con el lema "Pivote hacia Asia" el cual, por un lado, pareciera recordar la política de contención de Estados Unidos hacia Unión Soviética durante la época de la Guerra Fría y, por el otro, constituye una política cualitativamente nueva para la contención en el siglo XXI. A pesar de las iniciativas prometedoras de promover cooperación internacional dentro del marco del supergrupo G2 compuesto por los Estados Unidos y República Popular de China, las iniciativas de los EE.UU. se concentraron en los intentos de contrarrestar y contener la influencia de China en la zona. Las iniciativas económicas como el Acuerdo Transpacífíco TPP (abandonado por Estados Unidos después 
de la llegada de Donald Trump al poder) debía haber creado una zona con el liderazgo de los EE.UU., claramente, excluyendo a China.

Por otro lado, las iniciativas de seguridad como el Diálogo de Seguridad Cuadrilateral son una expresión de creciente intento de contener la influencia de China también con los medios políticos y alianzas militares. Al mismo tiempo, al reforzar las bases militares en aquellas zonas en donde se sitúa el Collar de perlas chino, ha permitido crear la posibilidad de bloquear, efectivamente, las rutas de exportación y abastecimiento de China y, además, contener una posible futura ampliación de la zona de influencia de China al este por el Pacífico o al oeste por el continente euroasiático y Océano Índico. Así mismo, las alianzas nuevas como EE.UU. - Vietnam ( aunque marcada por la historia de conflicto mutuo) y Malasia, como con aliados tradicionales, Japón, Corea del Sur (a pesar de las incertidumbres sobre la voluntad de EE.UU. de cumplir los compromisos militares) y Filipinas, solidifica la contención contra la influencia china, al tiempo que los tratados de cooperación militar y de seguridad están aumentado la capacidad de reacción y despliegue rápido de tropas estadounidenses y sus aliados en Asia-Pacífico.

Sin embargo, China ha sido asertiva al momento de eludir estos métodos de contención. La expansión de la estrategia marítima china, tanto a través de los proyectos como de la dimensión marítima de la Nueva Ruta de la Seda, como con la creciente presencia militar en las disputas territoriales en las zonas vitales para los intereses económicos, comerciales y estratégicos chinos en el Mar del Sur de China, está permitiendo un mayor posicionamiento del régimen de Pekín. De todas maneras, también aumenta los puntos de choque entre Estados Unidos y China con posibilidad de escalar los conflictos en el futuro.

\section{Referencias}

Acosta, J. A., Londoño, G., \& González, C. (2015). Pertinencia de los aportes teóricos para el análisis internacional desde la periferia. Escenarios: empresa y territorio, 171-193.

Actualidad RT. (27 de abril de 2016). "Ni petróleo, ni gas": la razón de la disputa en el mar de la China Meridional es otra. Actualidad RT. https://url2.cl/SV15E

Ahumada, E. (2008). Barack Obama y sus relaciones con el Asia Pacifico. Biblioteca del Congreso Nacional de Chile. https://url2.cl/DV7xD

Ajami, R. A. (2018). Geopolitics and Trade across Asia-Pacific Countries: Conflict or Convergence. Journal of Asia-Pacific Business, 43-45. 
Almoguera, P., \& Fontdeglória, X. (2018). Asia saca adelante el TPP y se pone al frente de la liberalización comercial. El País. https://n9.cl/zsu0v

Arancón, F. (2014). El collar de perlas de china: Geopolítica en el Índico. El Orden Mundial. https://elordenmundial.com/el-collar-de-perlas-chino/

Australian Government Department of Foreign Affairs and Trade. (2018). Regional Comprehensive Economic Partnership. Australian Government Department of Foreign Affairs and Trade. https://url2.cl/GbY6t

Bassets, L. (2012). El pivote asiático de Obama: La dificultad del momento queda expresada por el estallido de la crisis de Gaza. El País. https://n9.cl/tgyf

Batalla, X. (2006). Bipolar, unipolar, multipolar. 1983-2006: Los cambios más profundos. CIDOB Barcelona Centre for International Affairs.

BBC Mundo. (2017). Qué es el TPP, el gran acuerdo económico de 11 países del cual EE.UU. se acaba de retirar por orden de Donald Trump. BBC Mundo. https://url2.cl/Dqgs2

BBC News. (2014). Conflicto territorial desata ira anti-China en Vietnam. $B B C$. https://n9.cl/u5fm

Bergsten, F. C. (2009). Two’s Company. Foreign Affairs. https://www.foreignaffairs. com/articles/americas/2009-09-01/twos-company

Bustelo, P., \& Soto, A. (2003). Las relaciones entre Estados Unidos y China: ¿Asociación o competencia estratégicas? Real Instituto Elcano Royal Institute. https://bit.ly/3f9kUbq

Bustelo, P. (2004). Las relaciones entre Estados Unidos y China: ¿El final de la luna de miel? Real Instituto Elcano de Estudios Internacionales y Estratégicos. http://biblioteca.ribei.org/585/1/ARI-179-2004-E.pdf

Castro, G., Acosta, J., \& Praj, D. (2019). Aproximaciones al nuevo escenario mundial: Colombia ante la región de Asia Pacífico. Escenarios: empresa y territorio, 1-25.

Creus, N. (2013). El concepto de poder en las relaciones internacionales. Estudios Internacionales, 63-78. https://scielo.conicyt.cl/pdf/rei/v45n175/art03.pdf

Cuenca, A. (2018). Aguas revueltas en el mar de la China meridional. https://n9.cl/ $\mathrm{p} 9 \mathrm{cf}$

Dalton, M. (2020). Beyond Port Visits, US-Vietnam Relations Can Go Further. The Diplomat. https:/thediplomat.com/2020/03/ beyond-port-visits-us-vietnam-relations-can-go-further/

Dougherty, J., \& Pfaltzgraff, R. (1971). Contending theories of international relations. Philadelphia. 
DW. (2019). ASEAN summit: Asia trade bloc deal 'expected' in February 2020. $D W$.

Economy, E. C., \& Segal, A. (2009). The G-2 Mirage: Why the United States and China Are Not Ready to Upgrade Ties. Foreign Affairs. https://www.foreignaffairs.com/articles/east-asia/2009-05-01/g-2-mirage

El Orden Mundial. (2018). La geopolítica de China de Estados Unidos en Asia-Pacifico. El Orden Mundial. https:/elordenmundial.com/mapas/ china-y-estados-unidos-asia-pacifico/

Escudero, G. (2018). El Mar del Sur de China, nodo de la geopolítica regional y mundial del siglo XXI. Universidad de Granada. https://n9.cl/t7xt

Garrett, G. (2014). The G2 in the G-20: China, the United States, and the World after the Global Financial Crisis. Global Policy Journal. https://n9.cl/kdh5t

Giné, J. (2010). Barack Obama apuesta por Asia-Pacífico. Estudios de Política Exterior. https://n9.cl/g7a4h

Green, M., \& Goodman, M. (2015). After TPP: The Geopolitics of Asia and the Pacific. The Washington Quarterly.

Instituto de Estudios para la Paz de Estocolmo. (2018). SIPRI Yearbook 2018. Armaments, Disarmament, and International Security. https://www.sipri.org/ yearbook $/ 2018$

Mandelbaum, M. (Abril de 2019). The New Containment. Foreign Affairs. https:// n9.cl/gox0e

Manyin, M. E., Daggett, S., Dolven, B., Lawrence, S. V., Martin, M. F., O’Rourke, R., \& Vaughn, B. (2012). Pivot to the Pacific? The Obama Administration's "Rebalancing" Toward Asia. Federation of American Scientists. https://fas. org/sgp/crs/natsec/R42448.pdf

Martínez, J. C. (2018). Donald Trump y su influencia en Asia Pacifico. Observatorio Virtual Asia-Pacífico. https://n9.c1/32f9

Meick, E., \& Salidjanova, N. (2017). China's Response to U.S.-South Korean China's Response to U.S.-South Korean Implications. Staff Research Report. Obtenido de https://url2.cl/cyCrS

Mijares, V. M. (2006). Geoestrategia y Seguridad en el Mar de China Meridional. Universidad Central de Venezuela. https://n9.cl/khkq

Minh Tri, N. (19 de mayo de 2017). China's A2/AD Challenge in the South China Sea: Securing the Air from the Ground. The Diplomat. https://n9.cl/mauew

Morgenthau, H. (2003). A politica entre as naçoes. A luta pelo poder e pela paz. Instituto de Pesquisa de Relações Internacionais. 
Nye Jr., J. (2004). Soft Power: The Means to Success in World Politics. Public Affairs. UK.

Observatorio de la Política China. (2009). China considera ilegal propuesta de Vietnam sobre límites exteriores de plataforma continental. Observatorio de la Política China. https://n9.cl/cwy8

Pacheco, R. (2014). Return of the G2: Can US and China run the world? The Telegraph. https://n9.cl/jxh7

Panda, A. (2017 de agosto de 2017). How Much Trade Transits the South China Sea? Not \$5.3 Trillion a Year. The Diplomat. https://n9.cl/56ar.

Pérez, I. (1996). El realismo y el final de la Guerra Fría. Pontificia Universidad Católica del Perú.

Piqué, J. (2020 de febrero de 2020). Malaca: Centro de gravedad y campo de batalla. El Confidencial.

Portinario, P. P. (2000). Léxico de la politica. En L. Olamendi Baca, J. Liwerant Bokser, F. Castañeda, I. H. Cisneros, \& G. Fernández del Castillo Pérez. Fondo de Cultura Económica, Consejo Nacional de Ciencia y Tecnología, Fundación Heinrich Böll, Facultad Latinoamericana de Ciencias Sociales, Sede Académica de México.

Praj, D. (2018). Introducción a las Relaciones Internacionales. Esumer.

Praj, D., \& Restrepo, J. C. (2017). The Geopolitics of the Chinese Reform. Revista de Relaciones Internacionales, Estrategia y Seguridad.

Raffo, L., García, E., \& Díaz, V. (2018). La Alianza del Pacífico y los efectos potenciales del Acuerdo Transpacífico de Cooperación Económica sin Estados Unidos. Finanzas, Política y Economía

Rodríguez, I. (2014). Nuevas configuraciones económicas en el Asia-Pacífico y sus consecuencias en América Latina: Desde el APEC a la Alianza del Pacífico. DADOS-Revista de Ciências Sociais, 57, 553-580. https://www.redalyc.org/ $\mathrm{html} / 218 / 21831470009 /$

Sánchez, Y. (2017). La salida de Estados Unidos del TPP: Reacciones y posibles escenarios. Consejo Latinoamericano de Ciencas Sociales CLACSO. https:// core.ac.uk/download/pdf/83823325.pdf

Seligman, L. \& Gramer, R. (2019). Trump Presses Japan to Pay Up for U.S. Troops. Foreign Policy. https://foreignpolicy.com/2019/11/18/ trump-japan-south-korea-pay-united-states-troops-billions-asia-pacific/

Sempa, F. (2019). The Case for Containing China. The Diplomat. 
Sepúlveda, R. (2012). Estados Unidos y China -el Llamado G2- una legitimidad ficticia de dos potencias en declive. https://n9.cl/7143r

Sistema de Información sobre Comercio Exterior. (2013). Acuerdo de Asociación Transpacifico (TPP) - Australia, Brunei Darussalam, Canadá, Chile, Estados Unidos, Malasia, México, Nueva Zelandia, Perú, Singapur y Viet Nam. Foro Internacional Democracia y Cooperación. https://n9.cl/7j2k

Sistema de Información sobre Comercio Exterior. (2017). Acuerdo amplio y progresista de asociación transpacifico (CPTPP) - Australia, Brunei Darussalam, Canadá, Chile, Japón, Malasia, México, Nueva Zelandia, Perú, Singapur y Viet Nam. Sistema de Información sobre Comercio Exterior. https:// n9.cl/tndsg

Stashwick, S. (5 de mayo de 2018). China Deploys Long-Range Anti-Ship and AntiAir Missiles to Spratly Islands for First Time. The Diplomat.

Tellis, A. J. (2014). The geopolitics of the TTIP and the TPP. Adelphi Series, 93-120.

National Bureau of Statistics of China. (2017). The China National Statistics Yearbook. http://www.stats.gov.cn/tjsj/ndsj/2017/indexeh.htm

Vargas, L. (2019). China descubre gigantesca reserva de petróleo y gas. La República. https://n9.cl//rm58

Vuving, A. (6 de mayo de 2016). South China Sea: Who Occupies What in the Spratlys? The Diplomat. https://n9.cl/dhvae

Whipple, T. (2017). The End of the Oil Age. Escenarios: Empresa y territorio, 6, 129-144. 
